\title{
Astragaloside IV ameliorates necrotizing enterocolitis by attenuating oxidative stress and suppressing inflammation via the vitamin D3-upregulated protein 1/NF- $\kappa$ B signaling pathway
}

\author{
ZHIYONG CAI* , JINDI LIU*, HONGLIANG BIAN and JINLAN CAI
}

\author{
Newborn Department, Yancheng Maternity and Child Health Care Hospital, Yancheng, Jiangsu 224000, P.R. China
}

Received May 5, 2015; Accepted July 26, 2016

DOI: $10.3892 /$ etm.2016.3629

\begin{abstract}
Astragaloside IV (AS-IV) is a flavonoid from the plant Astragalus membranaceus (Fisch) Bge that has a wide range of therapeutic effects. The aim of the present study was to examine the effect of AS-IV on rats with necrotizing enterocolitis (NEC) under oxidative stress and inflammation. Newborn Sprague-Dawley rats were induced with NEC by asphyxia and hypothermia applied on 3 consecutive days. The rats were orally administered AS-IV at 25, 50 and $75 \mathrm{mg} / \mathrm{kg}$ for 4 days. The results revealed that AS-IV administration prevented NEC-induced decrease in the concentration of malondialdehyde and myeloperoxidase, and increase in the activity of glutathione (GSH) and superoxide dismutase in murine models. AS-IV also inhibited NEC-induced elevation in the levels of interleukin (IL)-6, IL-1 $\beta$, tumor necrosis factor- $\alpha$ and nuclear factor $(\mathrm{NF})-\kappa \mathrm{B}$. The effects of AS-IV were achieved under inflammation and oxidative stress. Western blotting demonstrated that AS-IV substantially inhibited the phosphorylated (p)-I $\kappa \mathrm{B} \alpha, \mathrm{NF}-\kappa \mathrm{Bp} 65, \mathrm{p}-\mathrm{NF}-\kappa \mathrm{Bp} 65$ protein levels and increased vitamin D3 upregulated protein 1 (VDUP1) and $\mathrm{I} \kappa \mathrm{B} \alpha$ protein levels. These data indicate that AS-IV may be effective in the protection of NEC-induced ileum degeneration by inhibiting the levels of inflammatory markers and oxidative stress via the regulation of the VDUP1/NF- $\mathrm{B}$ signaling pathway.
\end{abstract}

\section{Introduction}

Necrotizing enterocolitis (NEC) is a severe gastrointestinal tract disease that affects newborn babies. Despite years of investigation, the etiology remains unclear, and accepted

Correspondence to: Professor Hongliang Bian, Newborn Department, Yancheng Maternity and Child Health Care Hospital, 34 Yulong Road, Yancheng, Jiangsu 224000, P.R. China

E-mail: hlbian05@126.com

${ }^{*}$ Contributed equally

Key words: astragaloside IV, necrotizing enterocolitis, oxidative stress, inflammation, vitamin D3-upregulated protein $1 /$ nuclear factor- $\kappa \mathrm{B}$ pathway prevention and treatment strategies are lacking (1). The immature intestinal barrier function and immune/inflammatory responses have been partly linked to NEC (2). Loss of mucosal integrity due to a variety of factors (ischemia, infection and inflammation) and the host response to injury (circulatory, immunologic and inflammatory) are the leading causes of necrosis of the affected area (3). In infants with NEC, a compromised epithelial barrier and immature immune response can lead to exaggerated inflammation and oxidative stress damage. The excessive inflammatory and oxidative stress is implicated in the pathogenesis of NEC (4).

Vitamin D3-upregulated protein 1 (VDUP1) is a $46-\mathrm{kDa}$ intracellular protein that was initially isolated in HL-60 cells, and its expression is upregulated by vitamin D3 administration (5). VDUP1 interacts with the antioxidant thioredoxin; hence, it is considered to increase the vulnerability of cells to oxidative stress (6). VDUP1 is upregulated by various stresses, including $\mathrm{H}_{2} \mathrm{O}_{2}$, irradiation, heat shock, serum starvation and transforming growth factor- $\beta$ (7). Although deficits in antioxidants and increased oxidative stress accompanying NEC rat have been directly implicated in the pathogenesis, a possible role for VDUP1 in the adverse outcomes accompanying NEC has not yet been investigated.

The nuclear factor (NF)- $\kappa \mathrm{B}$ transcription factor family is a crucial mediator of the inflammatory process and is important in other processes, including cell development, growth, survival and proliferation. $\mathrm{NF}-\kappa \mathrm{B}$ can be activated by numerous stimuli including inflammatory factors, microbial molecules and genotoxic tension (8). Recent studies have demonstrated the pathological involvement of the immune system in NEC $(2,9)$. The levels of pro-inflammatory cytokines are increased in the intestines in NEC patients and animals (10). It has also been reported that pro-inflammation may be significant in the pathogenesis of NEC (11). In addition, correlative studies have demonstrated that antioxidants reduced NEC severity in in vivo experimental models (12). There is a close association between oxidative stress and inflammation in NEC and we hypothesize that an increase in oxidative stress-derived inflammation is one of the primary mechanisms underlying the pathogenesis and progression of NEC.

Astragaloside IV (AS-IV) is a major saponin purified from Astragalus membranaceus (Fisch) Bge, and has been widely used as a herbal prescription in traditional chinese medicine for its anti-inflammatory, antioxidative, carioprotective and 
immune-system stimulatory activities $(13,14)$. Recent evidence suggests that AS-IV is able to ameliorate ischemic brain injury, diabetic peripheral neuropathy, hepatic fibrosis and myocardial injury (15). In addition, AS-IV has been demonstrated to inhibit the pro-inflammatory transcriptional factor, $\mathrm{NF}-\kappa \mathrm{B}$, tumor necrosis factor (TNF) $-\alpha$ and mediate the $\mathrm{p} 38$ mitogen-activated protein kinase signaling pathway (16).

However, the beneficial effect of AS-IV on NEC remains to be investigated. In the present study, we hypothesized that AS-IV, which has protective effects in an NEC murine model, may also be associated with the inhibition of inflammation and antioxidative effects $(15,17)$. The present study was undertaken to evaluate the protective effects of AS-IVs and to elucidate the mechanism underlying these protective effects in rats.

\section{Materials and methods}

Materials. AS-IV (pure: 98\%) was purchased from the National Institutes for Food and Drug Control (Beijing, China). The enzyme-linked immunosorbent assay (ELISA) kits for determination of interleukin (IL)-6 (cat. no. R6000B), IL-1 $\beta$ (cat. no. RLB00) and TNF- $\alpha$ (cat. no. RTA00) were purchased from R\&D Systems (Minneapolis, MN, USA). The diagnostic kits for glutathione (GSH; cat. no. A006-2), malondialdehyde (MDA; cat. no. A003-1), superoxide dismutase (SOD; cat. no. A001-1) and myeloperoxidase (MPO; cat. no. A044) were purchased from Nanjing Jiancheng Bioengineering Institute (Nanjing, China). Primary antibodies for I $\mathrm{B} \mathrm{B} \alpha(1: 1,000$; mouse monoclonal; cat. no. 4814), phosphorylated (p)-IкB $\alpha$ (1:1,000; mouse monoclonal; cat. no. 9246), NF- $\mathrm{Bp} 65$ (1:1,000; mouse monoclonal; cat. no. 6956), p-NF-кBp65 (1:1,000; rabbit monoclonal; cat. no. 3039) and VDUP1 (1:1,000; rabbit monoclonal; cat. no. 14715) were purchased from Cell Signaling Technology, Inc. (Danvers, MA, USA). In addition, secondary antibodies were acquired from Santa Cruz Biotechnology, Inc. (Dallas, TX, USA).

Animals. Animal experiments were approved by the Institutional Animal Care and Use Committee, Nanjing Medical University (Nanjing, China). A total of 40 Sprague-Dawley murine neonates (15 days old, 20-35 g) were used in the present study and kept under controlled conditions. The murine neonates were kept with their mothers in indistinguishable cages (1 cage per group of 10 neonates). Tap water and standard rodent food supplement were used to feed the mothers. During the study period, the neonates were fed freely on breast milk by their mothers. The experimental animals were maintained in a controlled environment $\left(12: 12 \pm 1\right.$-h light/dark cycle; temperature, $22 \pm 3^{\circ} \mathrm{C}$; relative humidity, 55\%). Furthermore, the rats were allowed to acclimatize to the laboratory for $\geq 7$ days under climate-controlled conditions. The experiments were performed in adherence to the guidelines for the Care and Use of Laboratory Animals of the National Institute of Health.

Experimental design. Experimental NEC was induced by asphyxia (breathing 100\% nitrogen gas for $2 \mathrm{~min}$ ) and hypothermia $\left(4^{\circ} \mathrm{C}\right.$ for $\left.10 \mathrm{~min}\right)$ twice daily for 3 consecutive days. Age-matched normal rats served as a control. Neonatal rats were divided into the following experimental groups $(n=10$ for all groups): NEC control rats treated with saline solution for
4 days (group 1), NEC rats treated with $25 \mathrm{mg} / \mathrm{kg} / \mathrm{d}$ AS-IV for 4 days (group 2), NEC rats treated with $50 \mathrm{mg} / \mathrm{kg} / \mathrm{d}$ AS-IV for 4 days (group 3) and NEC rats treated with $75 \mathrm{mg} / \mathrm{kg} / \mathrm{d}$ AS-IV for 4 days (group 4). On the fourth day, the animals were sacrificed via decapitation, and the biochemical estimations were completed on the same day.

Histopathologic examination. To investigate the effects of AS-IV on the protection against NEC, hematoxylin and eosin staining (H\&E) was performed. Upon sacrifice, the intestines from the rats in all of the groups were resected. For each tissue, corresponding blocks of H\&E were scored (18). Pathological changes in intestinal architecture were evaluated using the NEC histological injury scoring system described by Nadler et al (19). The grading system was as follows: Grade 0, normal; grade 1 (mild), separation of villous core, without other abnormalities; grade 2 (moderate), villous core separation, submucosal edema and epithelial sloughing; and grade 3 (severe), denudation of epithelium with loss of villous, full-thickness necrosis or perforation.

GSH, MDA, SOD and MPO measurements. Partial distal ileum tissue samples were taken and rapidly homogenized with ice-cold normal saline, and the tissue homogenate $(10 \% \mathrm{w} / \mathrm{v})$ was prepared for further biochemical experiments. SOD activity was assessed using the xanthine oxidase method by using commercially available kits and MDA content was quantified using a thiobarbituric acid assay with 1,1,3, 3-tetramethoxypropane as an external standard at $532 \mathrm{~nm}$ according to the manufacturer's instructions. GSH activity was measured with a dithio-dinitrobenzoic acid kit at the absorbance of $412 \mathrm{~nm}$, and MPO activity was measured as previously described by Hillegass et al (20). Each measurement was performed in duplicate.

Measurement of cytokine levels. Serum TNF- $\alpha$, IL-1 $\beta$, and IL-6 concentration were determined from samples performed in parallel and repeated three times using ELISA kits according to the manufacturer's instructions. The optical density of each well was read at $450 \mathrm{~nm}$.

Quantification of mRNA expression. Total RNA was isolated from the intestinal tissue using TRIzol reagent (Invitrogen; Thermo Fisher Scientific, Inc., Waltham, MA, USA) according to the manufacturer's instructions. Next, the gene expression levels of IL-1 $\beta$, IL- $6, \mathrm{TNF}-\alpha$ and the $\mathrm{p} 65$ subunit of NF- $\kappa \mathrm{B}$ in the distal ileum were determined by reverse transcription-quantitative polymerase chain reaction (RT-qPCR) with the use of a PrimeScript RT Reagent kit (Takara Bio, Inc., Otsu, Japan), StepOnePlus Real-Time PCR system (Thermo Fisher Scientific Inc.) and SYBR Premix Ex Taq II (Takara Bio, Inc.). The primers used have been reported previously $(21,22)$. The specific PCR primer pairs (purchased from GenScript Corporation, Piscataway Township, NJ, USA) for the target genes were as follows: Glyceraldehyde 3-phosphate dehydrogenase forward, 5'-CCATGGAGAAGGCTGGGG-3' and reverse, 5'-CAAAGT TGTCATGGATGACC-3'; NF- $\kappa$ B forward, 5'-CATTGAGGT GTATTTCACGG-3' and reverse, 5'-GGCAAGTGGCCATTG TGTTC-3'; IL-6 forward, 5'-GGAGTTCCGTTTCTACCT GG-3' and reverse, 5'-GCCGAGTAGACCTCATAGTG-3'; TNF- $\alpha$ forward, 5'-CCACGTCGTAGCAAACCACCAAG-3' 
and reverse, 5'-CAGGTACATGGGCTCATACC-3'; IL-1 $\beta$ forward, 5'-TTGGGATCCACACTCTCCAG-3' and reverse, 5'-AGAAGCTGTGGCAGCTACCT-3. The following thermal cycling conditions were performed: $95^{\circ} \mathrm{C}$ for $10 \mathrm{~min} ; 95^{\circ} \mathrm{C}$ for $15 \mathrm{sec} ; 60^{\circ} \mathrm{C}$ for $30 \mathrm{sec}$; and $72^{\circ} \mathrm{C}$ for $30 \mathrm{sec}$ for 40 cycles. Samples were quantified according to the $2^{-\Delta \Delta \mathrm{Cq}}$ method (23).

Western blotting of the VDUPI/NF- $\kappa B$ signaling pathway. Distal ileum tissue samples were homogenized using radioimmunoprecipitation buffer (Beyotime Institute of Biotechnology, Haimen, China) and centrifuged $\left(12,000 \mathrm{x} \mathrm{g}, 20 \mathrm{~min}, 4^{\circ} \mathrm{C}\right)$ for western blot analysis. Equal amounts $(50 \mu \mathrm{g})$ of protein were separated by a $10 \%$ SDS-PAGE gel, and transferred onto a polyvinylidene difluoride membrane using standard procedures (Bio-Rad Laboratories, Inc., Hercules, CA, USA). Following blocking with $1 \%$ bovine serum albumin (Beyotime Institute of Biotechnology) in phosphate-buffered saline for $1.5 \mathrm{~h}$ at $37^{\circ} \mathrm{C}$, the blots were incubated for $1 \mathrm{~h}$ with the appropriate concentration of specific antibody at $37^{\circ} \mathrm{C}$, followed by horseradish peroxidase-conjugated anti-rabbit (1:5,000; cat. no. sc-2030) or anti-mouse (1:5,000; cat. no. sc-2302) secondary antibody for $1 \mathrm{~h}$ at $37^{\circ} \mathrm{C}$. After the bands were washed, the immune-reactive proteins were visualized by enhanced chemiluminescence reagent (EMD Millipore, Billerica, MA USA). The band intensities were quantified using the ChemiDoc MP system (Bio-Rad Laboratories, Inc.).

Statistical analysis. Data are reported as means \pm standard error of the mean. Statistical analysis of the results was performed by one-way analysis of variance followed by Fisher's protected least significant difference using the statistical program StatView for Macintosh computers (Abacus Concepts, Berkeley, CA, USA). $\mathrm{P}<0.05$ was considered to indicate a statistically significant difference.

\section{Results}

Effect of AS-IV on the incidence of NEC. In the present study, the severity of the histological changes of the ileal segments and the incidence of NEC in all of the groups were determined using a scoring system between 0 and 3 . The histologic grading of intestinal injury is presented in Table I. In the normal control group, no evident histological alteration was observed in the ileal specimens. By contrast, ileal tissue samples obtained from the NEC group rats demonstrated significant pathological changes, such as pneumatosis intestinalis, fragility, edema, discoloration and weakness of tissue integrity. However, NEC rat pathological changes were significantly ameliorated by AS-IV as demonstrated in the representative images, particularly at a high dose (75 mg/kg; Fig. 1).

Antioxidant parameters. The GSH levels in the NEC group were significantly lower, as compared with the controls $(\mathrm{P}=0.0004)$. Following treatment with AS-IV, the GSH levels in three treatment groups were increased. The $75 \mathrm{mg} / \mathrm{kg}$ group was closest to the mean value in the normal control group, which represents a significant increase in the GSH levels ( $\mathrm{P}=0.0094$; Fig. 2A).

In the NEC group, the tissue SOD activity was significantly increased compared with the normal group ( $\mathrm{P}=0.0088$; Fig. 2B). In comparison with the model rats, AS-IV supplementation in
Table I. Comparison of histopathological features in each group.

\begin{tabular}{lc} 
Group & Intestinal injury s \\
\hline Control $(\mathrm{n}=12)$ & 0 \\
NEC $(\mathrm{n}=7)$ & $2.6 \pm 0.53^{\mathrm{a}}$ \\
NEC + AS-IV $(25 \mathrm{mg} / \mathrm{kg} ; \mathrm{n}=8)$ & $2.1 \pm 0.83$ \\
NEC + AS-IV $(50 \mathrm{mg} / \mathrm{kg} ; \mathrm{n}=10)$ & $1.9 \pm 0.87^{\mathrm{b}}$ \\
NEC + AS-IV $(75 \mathrm{mg} / \mathrm{kg} ; \mathrm{n}=10)$ & $1.4 \pm 0.51^{\mathrm{c}}$
\end{tabular}

Values are presented as the mean \pm standard error of the mean in each group ( $\mathrm{n}=7-12$ ). ${ }^{\mathrm{a}} \mathrm{P}<0.01$, vs. the control; ${ }^{\mathrm{b}} \mathrm{P}<0.05,{ }^{\mathrm{c}} \mathrm{P}<0.01$, vs. the model group. NEC, necrotizing enterocolitis; AS-IV, astragaloside IV.

NEC rats prevented an increase in SOD levels, and the values in the AS-IV-treated NEC rats were significantly reduced compared with the model rats in a dose-dependent manner.

As shown in Fig. 2C, in the model group MDA levels were significantly increased, and this effect was gradually recovered by AS-IV treatment; the AS-IV group presented the lowest MDA levels compared with the dose treatments in the $75 \mathrm{mg} / \mathrm{kg}$ dose group ( $\mathrm{P}=0.0087)$. Furthermore, MPO levels were elevated by $>85 \%$ ( $P=0.0097$; Fig. 2D) in the NEC group compared to the age-matched normal control rats.

Inflammatory parameters. As illustrated in Fig. 3, TNF- $\alpha$ $(\mathrm{P}=0.0098), \mathrm{IL}-1 \beta(\mathrm{P}=0.0031)$ and IL-6 $(\mathrm{P}=0.004)$ levels were observed to be significantly increased in the NEC group compared with the normal group. AS-IV (50, and $75 \mathrm{mg} / \mathrm{kg}$ ) pretreatment efficiently reduced the production of TNF- $\alpha(\mathrm{P}=0.042$ and $\mathrm{P}=0.0112$, respectively), $\mathrm{IL}-1 \beta(\mathrm{P}=0.0086$ and $\mathrm{P}=0.0065$, respectively) and IL- $6(\mathrm{P}=0.0243$ and $\mathrm{P}=0.0083$, respectively) in a dose-dependent manner. At the mRNA level, the markedly increased transcripts of TNF- $\alpha$, IL- $1 \beta$, IL- 6 and $\mathrm{NF}-\kappa \mathrm{B}$ were detected in the distal ileum from the NEC model rats, whereas AS-IV administration significantly reversed these effects. Furthermore, the TNF- $\alpha$ value in the distal ileum of NEC rats was increased $\sim 2$-fold higher compared with the untreated normal distal ileum of the control group $(\mathrm{P}=0.0026)$ as well as the IL-1 $\beta$ ( $\mathrm{P}=0.0073$; Fig. $4 \mathrm{~A}$ and $\mathrm{B})$. In addition, IL-6 was elevated by $>2.7$ fold in the distal ileum that was obtained from NEC rats ( $\mathrm{P}=0.00069$; Fig. 4C). In addition, the results demonstrated that NF- $\mathrm{kB}$ values (Fig. 4D) in the AS-IV-treated rats were significantly lower compared with the untreated NEC. Differences were significant between the 25 and $75 \mathrm{mg} / \mathrm{kg}$ treated groups $(\mathrm{P}=0.0312)$.

Effect of AS-IV on the VDUPI/NF- $\kappa B$ signaling pathway. In order to investigate the change in VDUP1/NF- $\mathrm{KB}$ pathway protein expression in the distal ileum following AS-IV treatment, the proteins involved were examined by western blotting. As shown in Fig. 5, the phosphorylation of $\mathrm{I} \kappa \mathrm{B} \alpha$ and NF- $\mathrm{KB}$ was significantly upregulated in the model group $(\mathrm{P}=0.0056$ and $\mathrm{P}=0.0073$, respectively). In the AS-IV groups, the protein expression levels of $\mathrm{p}-\mathrm{I} \kappa \mathrm{B} \alpha, \mathrm{NF}-\kappa \mathrm{B}$ p 65 and $\mathrm{p}-\mathrm{NF}-\kappa \mathrm{B}$ were significantly reduced in comparison with the model group. In the AS-IV groups, the protein expression levels of VDUP1 and 

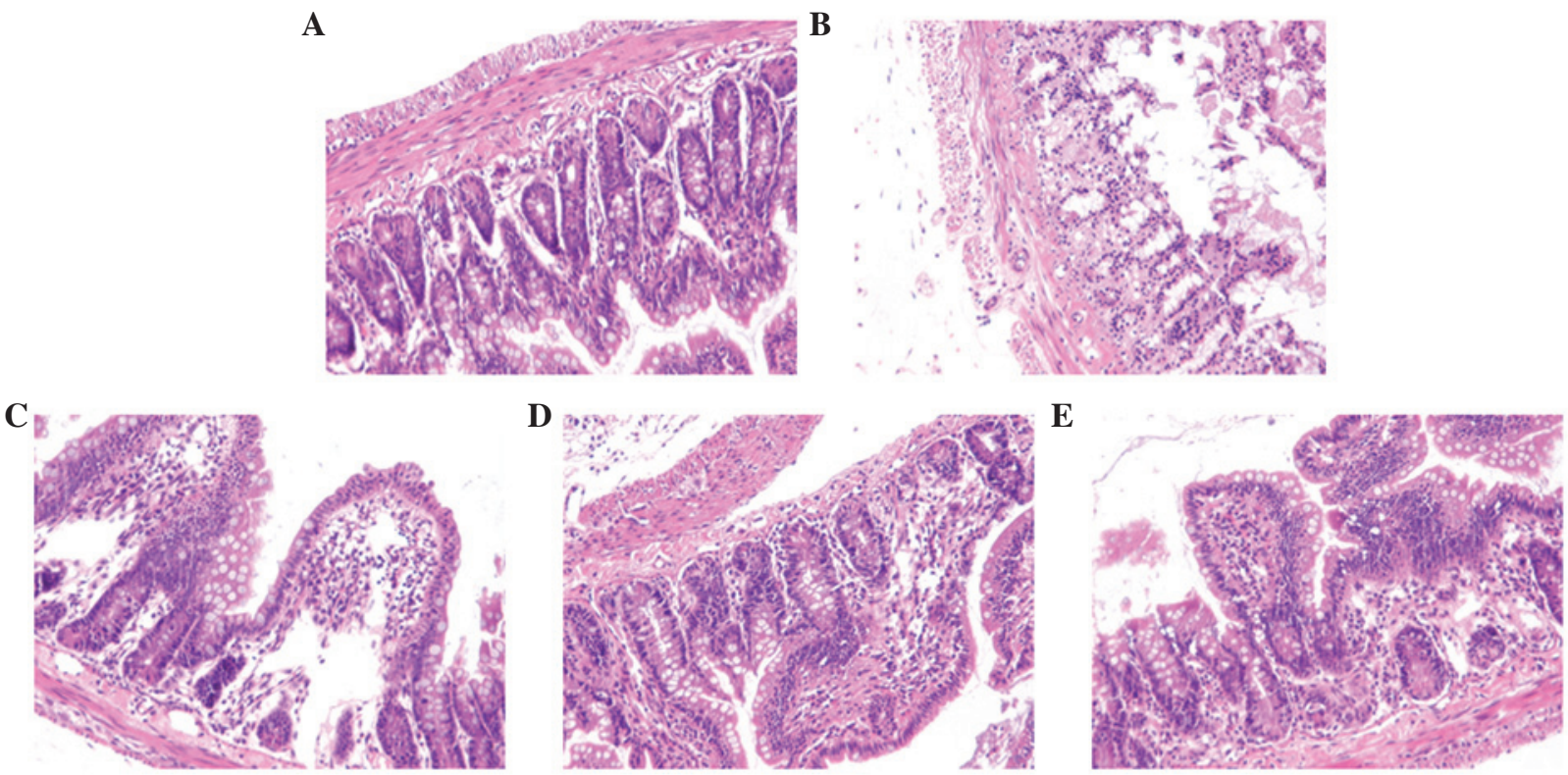

Figure 1. Representative histopathologic condition of the terminal ileum from each experimental group stained with H\&E (original magnification, $\mathrm{x} 10$ ). Intestinal architecture of a rat from (A) the control group revealed normal histological findings; (B) NEC group and NEC group treated with (C) 25, (D) 50 and (E) $75 \mathrm{mg} / \mathrm{kg}$ AS-IV groups. H\&E, hematoxylin and eosin; NEC, necrotizing enterocolitis; AS-IV, astragaloside IV.
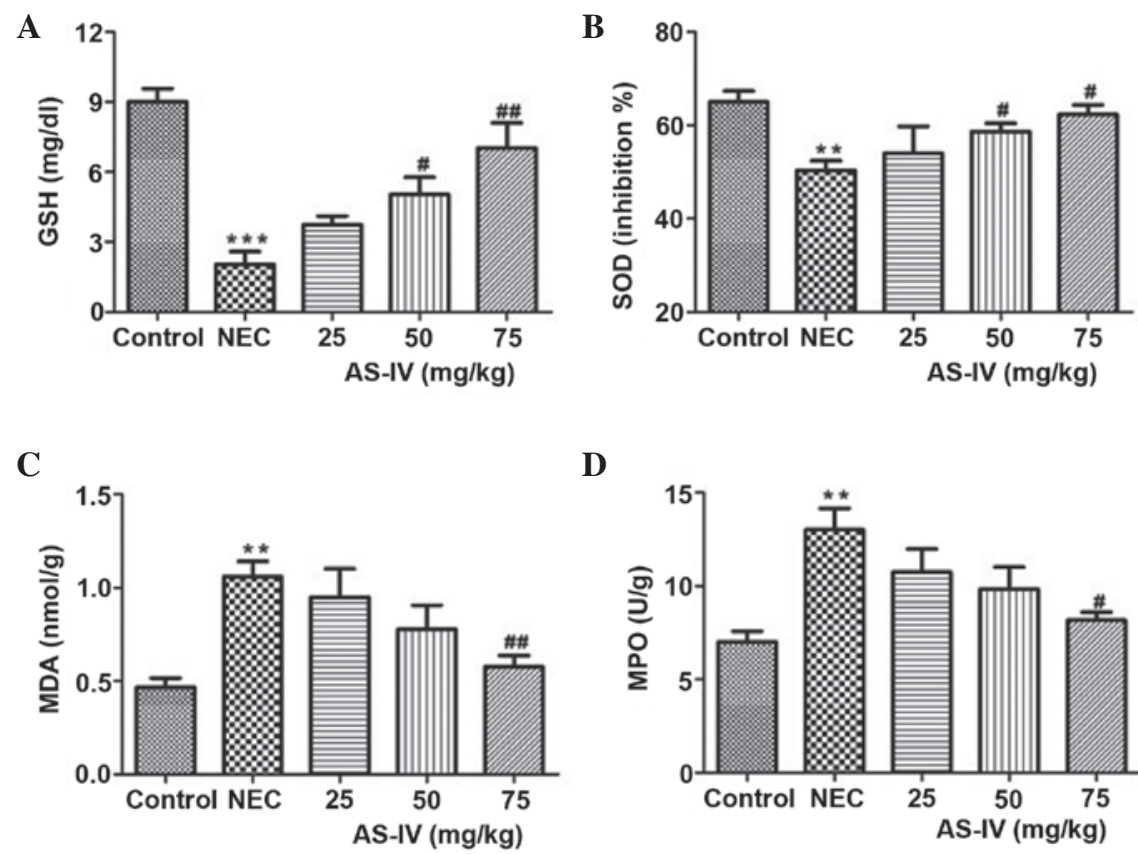

D

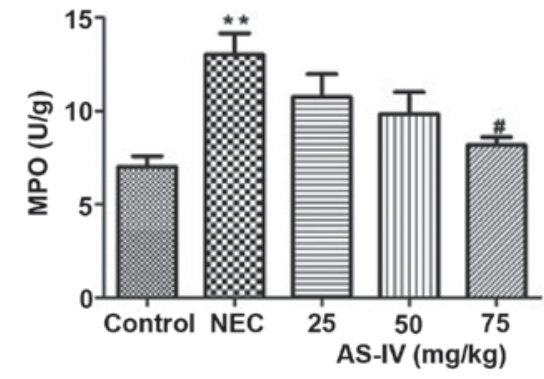

Figure 2. (A) GSH, (B) SOD, (C) MDA and (D) MPO concentrations in the distal ileum of rats after 4 days of treatment. Data are presented as the mean \pm standard error of the mean in each group $(\mathrm{n}=10),{ }^{{ }^{* *} \mathrm{P}}<0.01,{ }^{* * *} \mathrm{P}<0.001$, vs. the control group; ${ }^{*} \mathrm{P}<0.05,{ }^{\# \#} \mathrm{P}<0.01$, vs. the model group. GSH, glutathione; SOD superoxide dismutase; MDA, malondialdehyde; MPO, myeloperoxidase; NEC, necrotizing enterocolitis; AS-IV, astragaloside IV.

I $\kappa \mathrm{B} \alpha$ were significantly increased compared with the model group, particularly the high dose group $(\mathrm{P}=0.049$ and $\mathrm{P}=0.0039$, respectively).

\section{Discussion}

To the best of our knowledge, the present study is the first report demonstrating that AS-IV has beneficial effects on an NEC animal rat model, including on oxidative stress and inflammation, which are considered to be important in the intestines during NEC development. The present study illustrated the potential protective effects of AS-IV on NEC rats by attenuating oxidative stress and suppressing inflammation. Its mechanism of action may be via the regulation of the VDUP1/NF- $\mathrm{B}$ signaling pathway.

Oxidative stress, which has a negative effect and is caused by free radicals produced in vivo, is the keystone in multiple lines of evidence converging on the development and genesis of alimentary system disorders. It can cause profound damage to the intestines through dysregulation of the intracellular 

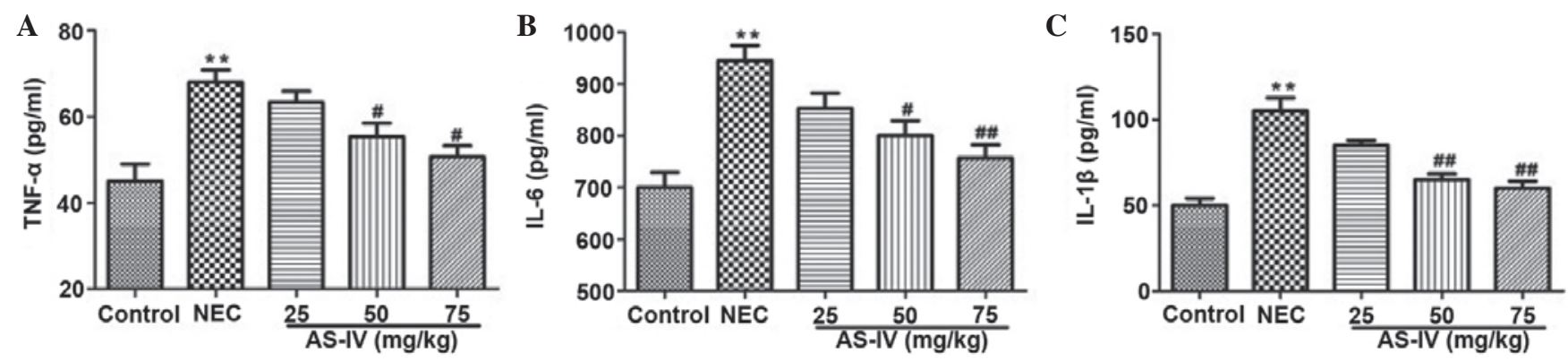

Figure 3. Effect of AS-IV on inflammatory cytokine production in the serum. The levels of (A) TNF- $\alpha$, (B) IL-1 $\beta$ and (C) IL-6 were measured by ELISA kits after 4 days of treatment. Data are presented as the means \pm standard error of the mean in each group $(\mathrm{n}=10),{ }^{* *} \mathrm{P}<0.01$, vs. the control group; ${ }^{\#} \mathrm{P}<0.05,{ }^{\# /} \mathrm{P}<0.01$, vs. the model group. AS-IV, astragaloside IV; TNF- $\alpha$, tumor necrosis factor- $\alpha$; IL-interleukin; NEC, necrotizing enterocolitis.

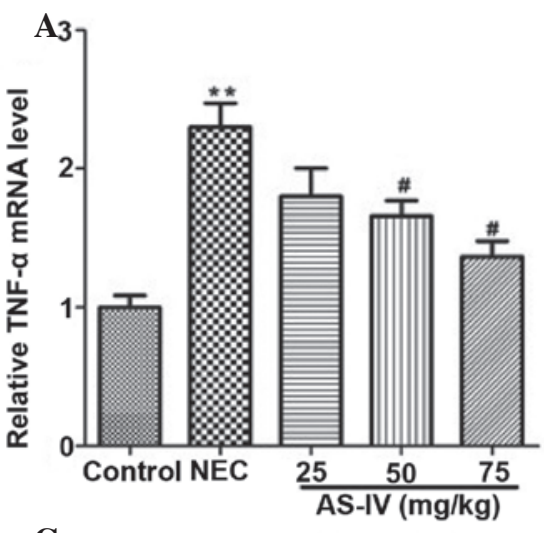

C

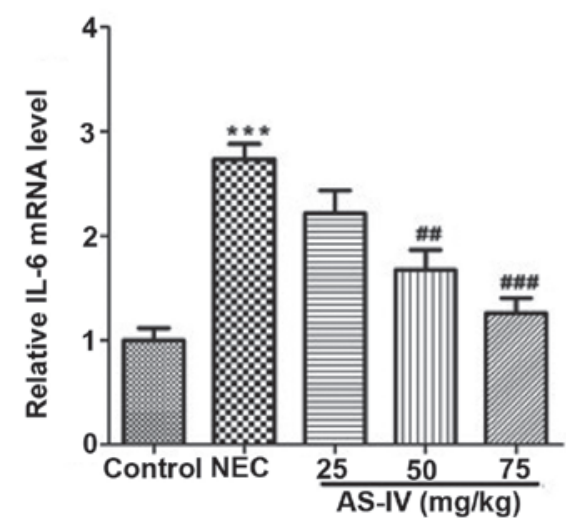

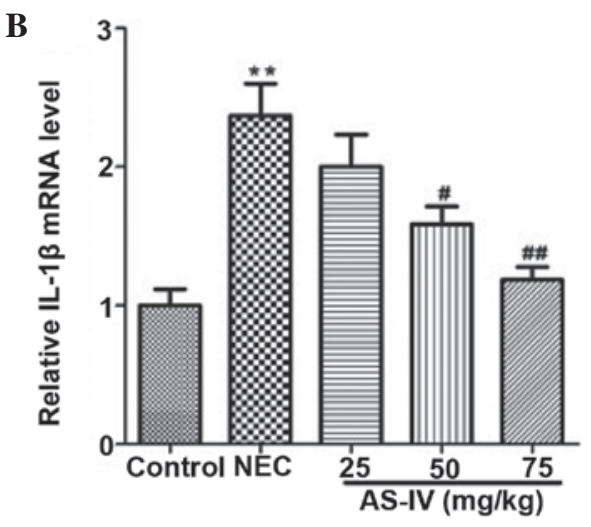

$\mathbf{D}$

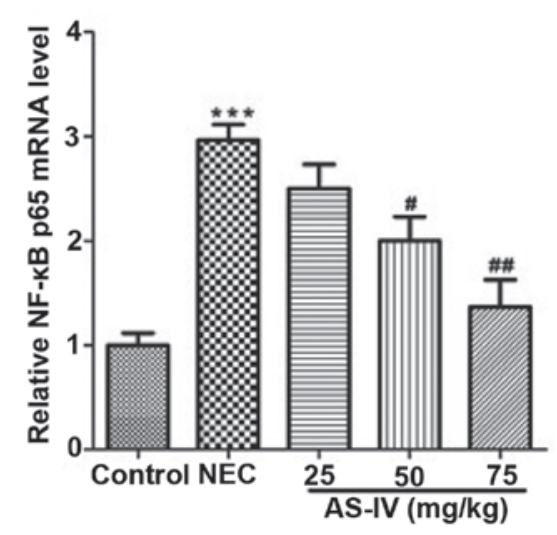

Figure 4. The mRNA expression levels of (A) TNF- $\alpha$, (B) IL-1 $\beta$ and (C) IL-6 and (D) NF- $\mathrm{kB}$ in rat distal ileum after 4 days treatment was detected by reverse-transcription polymerase chain reaction. Data represent the mean \pm standard error of the mean in each group $(\mathrm{n}=5)$, ${ }^{* *} \mathrm{P}<0.01$ and ${ }^{* * *} \mathrm{P}<0.001$, vs. the control group; ${ }^{\#} \mathrm{P}<0.05,{ }^{\# \#} \mathrm{P}<0.01$ and ${ }^{\# \# \#} \mathrm{P}<0.001$, vs. the model group. TNF- $\alpha$, tumor necrosis factor- $\alpha$; NEC, necrotizing enterocolitis; AS-IV, astragaloside IV; IL-interleukin; NF- $\kappa \mathrm{B}$, nuclear factor- $\mathrm{kB}$.

physiology leading to disorders. Clinical evidence suggests a systemic activation of the inflammatory response (24). Furthermore, recent studies have demonstrated samples from patients with NEC demonstrated elevated levels of various inflammatory mediators compared with gestational age-matched controls $(25,26)$. In addition, the antioxidant capacity of AS-IV has been considered to be confirm via its beneficial effects on the antioxidant defense system. The results of the present study indicated that after 4 days administration of 25, 50 and $75 \mathrm{mg} / \mathrm{kg}$ AS-IV, the levels of MDA and MPO were significantly decreased, with a more evident decrease following the administration of $75 \mathrm{mg} / \mathrm{kg}$ AS-IV. In addition, various doses of AS-IV could improve the levels of GSH and SOD in NEC rats, particularly at high doses. This suggests that AS-IV has the potential to inhibit the overall oxidative damage undergone by the intestines in NEC. In conclusion, the data of the present study suggest that AS-IV therapy may protect the intestine against NEC by modulating the oxidative/antioxidative status.

Inflammation is a common event that drives the development of various intestinal changes in patients with NEC. The pathophysiology of NEC involves a complex interaction of inflammatory mediators. The results of previous studies demonstrated the importance of inflammation in intestinal apoptosis and in metabolic memory, and pinpoints 

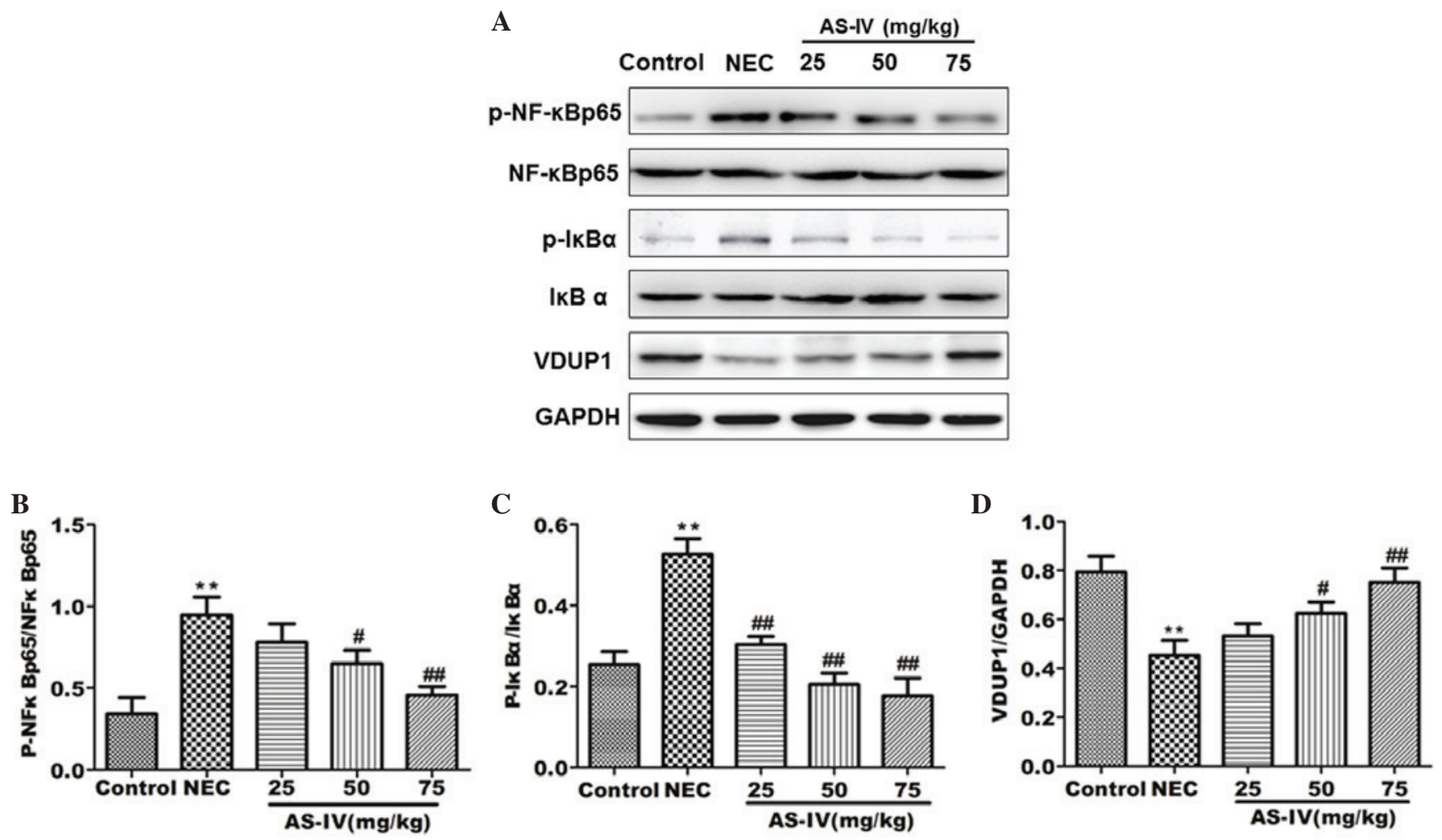

Figure 5. Protein expression levels in rat distal ileum after 4 days treatment were detected by (A) western blot analysis. The relative expressions of (B) p-NF-kB/ NF- $\kappa B$, (C) $\mathrm{p}-\mathrm{I} \kappa \mathrm{B} \alpha / \mathrm{I} \kappa \mathrm{B} \alpha$ and (D) VDUP1 were normalized to GAPDH. Data are presented as the means \pm standard error of the mean in each group (n=3),

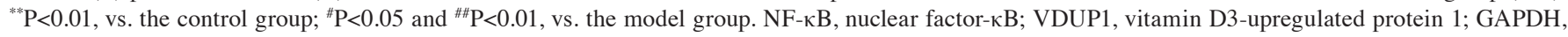
glyceraldehyde 3-phosphate dehydrogenase; NEC, necrotizing enterocolitis; AS-IV, astragaloside IV; p, phosphorylated.

the importance of the duration of the reversal in its outcome $(27,28)$. NEC has been demonstrated to upregulate various pro-inflammatory mediators during pathogenesis in the intestines, including IL-1 $\beta$, IL-6, TNF- $\alpha$, NF- $\mathrm{KB}$ and localized inflammatory processes that are considered to be important in the development of NEC (25). In the present study the levels of TNF- $\alpha$, IL- $1 \beta$ and IL- 6 in the serum were increased compared with the control group. In addition, the mRNA expression levels of TNF- $\alpha$, IL- $1 \beta$, IL- 6 and NF- $\kappa B$ were determined in the tissue of the intestine. However, the AS-IV-treated distal ileum revealed significantly lower levels of cytokines compared with the non-treated distal ileum.

AS-IV has been widely studied for its anti-inflammatory properties in rats (29). In the present study, three doses of AS-IV (25, 50 and $75 \mathrm{mg} / \mathrm{kg}$ body weight) were used. All the doses demonstrated potential protective effects against NEC. However, the $75 \mathrm{mg} / \mathrm{kg}$ dose exhibited a marked response against the anti-oxidant and anti-inflammatory parameters, though effects were comparable with the $25 \mathrm{mg} / \mathrm{kg}$ body weight-treated group on the remaining parameters. However, the effects were comparable with those of the $25 \mathrm{mg} / \mathrm{kg}$ dose in terms of the IL- $1 \beta$, IL- 6 , TNF- $\alpha$ and NF- $\mathrm{KB}$ expression levels. Furthermore, since a lower dose may induce less potential adverse events, more studies are required to investigate this low dose of AS-IV.

To further characterize the mechanism underlying the AS-IV protective effect on NEC rats, the effects of AS-IV on the activation of the VDUP1/NF- $\kappa \mathrm{B}$ signaling pathways were examined. As observed in the results, the level of VDUP1 was nearly recovered to the normal level following AS-IV treatment in the $75 \mathrm{mg} / \mathrm{kg}$ dose group. Furthermore, the levels of phosphorylation of NF- $\kappa \mathrm{Bp} 65$ and $\mathrm{I} \kappa \mathrm{B} \alpha$ were evidently increased in the model group, and administration of AS-IV impaired phosphorylation of these molecules in a dose-dependent manner. Furthermore, the present results demonstrated that AS-IV was able to significantly regulate the VDUP1/NF- $\kappa B$ signaling pathway in order to protect NEC rats.

In conclusion, AS-IV has beneficial effects in experimental studies of the NEC rats, which are characterized by increased oxidative stress and inflammatory reactions, supporting its clinical use. To the best of our knowledge, the present study is the first to demonstrate that AS-IV therapy has beneficial effects on rat NEC-like injuries by reducing the levels of inflammatory cytokines and improving antioxidant defense. The present results also demonstrated that AS-IV significantly regulated the VDUP1/NF- $\mathrm{kB}$ signaling pathway. Thus, AS-IV appears to be a useful adjunct therapy to possibly inhibit the development/progression of NEC, and the lethal complication faced by patients with NEC.

\section{Acknowledgements}

The present study was supported in part by grants from the Southeast University of China. The authors gratefully acknowledge associate Professor Su Ning for her assistance in the histopathological analysis. 


\section{References}

1. Chen CC and Allan WW: Probiotics and the mechanism of necrotizing enterocolitis. Semin Pediatr Surg 22: 94-100, 2013.

2. Nanthakumar N, Meng D, Goldstein AM, Zhu W, Lu L, Uauy R, Llanos A, Claud EC and Walker WA: The mechanism of excessive intestinal inflammation in necrotizing enterocolitis: An immature innate immune response. PLoS One 6: e17776, 2011.

3. Schnabl KL, Van Aerde JE, Thomson AB and Clandinin MT: Necrotizing enterocolitis: A multifactorial disease with no cure. World J Gastroenterol 14: 2142-2161, 2008.

4. Chandler JC and Hebra A: Necrotizing enterocolitis in infants with very low birth weight. Semin Pediatr Surg 9: 63-72, 2000.

5. Chung JW, Jeon JH, Yoon SR and Choi I: Vitamin D3 upregulated protein 1 (VDUP1) is a regulator for redox signaling and stress-mediated diseases. J Dermatol 33: 662-669, 2006.

6. Pan CL and Dong ZW: Antiasthmatic effects of eugenol in a mouse model of allergic asthma by regulation of vitamin D3 upregulated protein $1 / \mathrm{NF}-x \mathrm{~B}$ pathway. Inflammation 38: 1385-1393, 2015.

7. Kim SY, Suh HY, Chuang JW, Yoon SR and Choi I: Diverse functions of VDUP1 in cell proliferation, differentiation and diseases. Cell Mol Immunol 4: 345-351, 2007.

8. Kutuk $\mathrm{O}$ and Basaga $\mathrm{H}$ : Inflammation meets oxidation: $\mathrm{NF}-x \mathrm{~B}$ as a mediator of initial lesion development in atherosclerosis. Trends Mol Med 9: 549-557, 2003.

9. Tanner SM, Berryhill TF, Ellenburg JL, Jilling T, Cleveland DS, Lorenz RG and Martin CA: Pathogenesis of necrotizing enterocolitis: Modeling the innate immune response. Am J Pathol 185: 4-16, 2015.

10. Shilan M and Mohammad A: A review on the role of oxidative stress and inflammation in necrotizing enterocolitis and benefits of the phosphodiesterase inhibitor pentoxifylline. International Journal of Pharmacology 9: 245-250, 2013.

11. Frost BL, Jilling $\mathrm{T}$ and Caplan MS: The importance of pro-inflammatory signaling in neonatal necrotizing enterocolitis. Semin Perinatol 32: 100-106, 2008.

12. Cevik M, Karadag CA, Sakiz DE, Tander B and Embleton DD: The role of nitric oxide in an experimental necrotising enterocolitis model. Afr J Paediatr Surg 10: 24-28, 2013.

13. He Y, Du M, Gao Y, Liu H, Wang H, Wu X and Wang Z: Astragaloside IV attenuates experimental autoimmune encephalomyelitis of mice by counteracting oxidative stress at multiple levels. PLoS One 8: e76495, 2013.

14. Lai PK, Chan JY, Cheng L, Lau CP, Han SQ, Leung PC, Fung KP and Lau CB: Isolation of anti-inflammatory fractions and compounds from the root of Astragalus membranaceus. Phytother Res 27: 581-587, 2013.

15. Ren S, Zhang H, Mu Y, Sun M and Liu P: Pharmacological effects of Astragaloside IV: A literature review. J Tradit Chin Med 33: 413-416, 2013.
16. Chen Z, Cai Y,Zhang W, Liu X and Liu S: Astragaloside IV inhibits platelet-derived growth factor-BB-stimulated proliferation and migration of vascular smooth muscle cells via the inhibition of p38 MAPK signaling. Exp Ther Med 8: 1253-1258, 2014.

17. Yazıcı S, Akşit H, Korkut O, Sunay B and Çelik T: Effects of boric acid and 2-aminoethoxydiphenyl borate on necrotizing enterocolitis. J Pediatr Gastroenterol Nutr 58: 61-67, 2014.

18. Li J, Gu T, Fu X and Zhao R: Effect of salvianolic acid A and C compatibility on inflammatory cytokines in rats with unilateral ureteral obstruction. J Tradit Chin Med 35: 564-570, 2015.

19. Nadler EP, Dickinson E, Knisely A, Zhang XR, Boyle P, Beer-Stolz D, Watkins SC and Ford HR: Expression of inducible nitric oxide synthase and interleukin-12 in experimental necrotizing enterocolitis. J Surg Res 92: 71-77, 2000.

20. Hillegass LM, GriswoldDE,Brickson B and Albrightson-Winslow C: Assessment of myeloperoxidase activity in whole rat kidney. J Pharmacol Methods 24: 285-295, 1990.

21. Lu HE, Chen Y, Sun XB, Tong B and Fan XH: Effects of luteolin on retinal oxidative stress and inflammation in diabetes. RSC Advances 5: 4898-4904, 2015.

22. Williams CM and Coleman JW: Induced expression of mRNA for IL-5, IL-6, TNF-alpha, MIP-2 and IFN-gamma in immunologically activated rat peritoneal mast cells: Inhibition by dexamethasone and cyclosporin A. Immunology 86: 244-249, 1995.

23. Livak KJ and Schmittgen TD: Analysis of relative gene expression data using real-time quantitative PCR and the $2^{-\Delta \Delta \mathrm{Ct}}$ method. Methods 25: 402-408, 2001.

24. Lasanianos NG, Kanakaris NK, Dimitriou R, Pape HC and Giannoudis PV: Second hit phenomenon: Existing evidence of clinical implications. Injury 42: 617-629, 2011

25. Qi W, Shen Q, Zhang L, Han LP and Wang S: Study on the inflammatory intervention of erythropoietin on NEC. Exp Ther Med Jun 11: 2221-2224, 2016.

26. Heida FH, Hulscher JB, Schurink M, van Vliet MJ, Kooi EM, Kasper DC, Pones M, Bos AF and Benkoe TM: Bloodstream infections during the onset of necrotizing enterocolitis and their relation with the pro-inflammatory response, gut wall integrity and severity of disease in NEC. J Pediatr Surg 50:1837-1841, 2015.

27. Chung CY, Park YL, Kim N, Oh HH, Myung DS, Kim JS, Cho SB, Lee WS, Kim HS, Ahn BW and Joo YE: Rice prolamin extract ameliorates acute murine colitis by inhibiting nuclear factor-kappaB and modulating intestinal apoptosis and cell proliferation. Clin Exp Immunol 178: 537-547, 2014

28. Everard A, Geurts L, Caesar R, Van Hul M, Matamoros S, Duparc T, Denis RG, Cochez P, Pierard F, Castel J, et al: Intestinal epithelial MyD88 is a sensor switching host metabolism towards obesity according to nutritional status. Nat Commun 5: 5648, 2014.

29. Lu M, Tang F, Zhang J, Luan A, Mei M, Xu C, Zhang S, Wang H and Maslov LN: Astragaloside IV attenuates injury caused by myocardial ischemia/reperfusion in rats via regulation of toll-like receptor 4/nuclear factor- $\kappa \mathrm{B}$ signaling pathway. Phytother Res 29: 599-606, 2015. 